\title{
Ingested Pebbles causing Abdominal Radio-Opaque Shadows
}

Sir,

We present a plain abdominal radiograph of 3 years and 7 months old boy with history of pica showing multiple small rounded radio-opaque shadows in sigmoid colon and descending colon. Similar shadows were seen in part of transverse and ascending colon. This boy had ingested small pebbles over a period of few days and presented with history of pain abdomen, abdominal distension and passing small pebbles in stools. He was referred to the department of surgery, Government medical college, Jammu where gradual per rectal manual evacuation of pebbles was done over a period of few days.

When the plain abdominal radiograph shows multiple radio-opaque shadows, the possible differential diagnosis include multiple gall stones, urinary stones or multiple calcified lymphnodes, teratoma of mesentery, mesenteric fat necrosis and enteroliths. ${ }^{1}$ In a child with such a radiograph, possibility of ingested foreign bodies must be kept in mind. Foreign body ingestion is seen in children of all age groups and there is a tendency in small children to ingest foreign material in curiosity. Thus, the incidence of foreign body is greatest in children aged 6 months to 4 years. $^{2-3}$

A similar abdominal radiograph may be seen in an adult and rarely in older child having enteroliths.

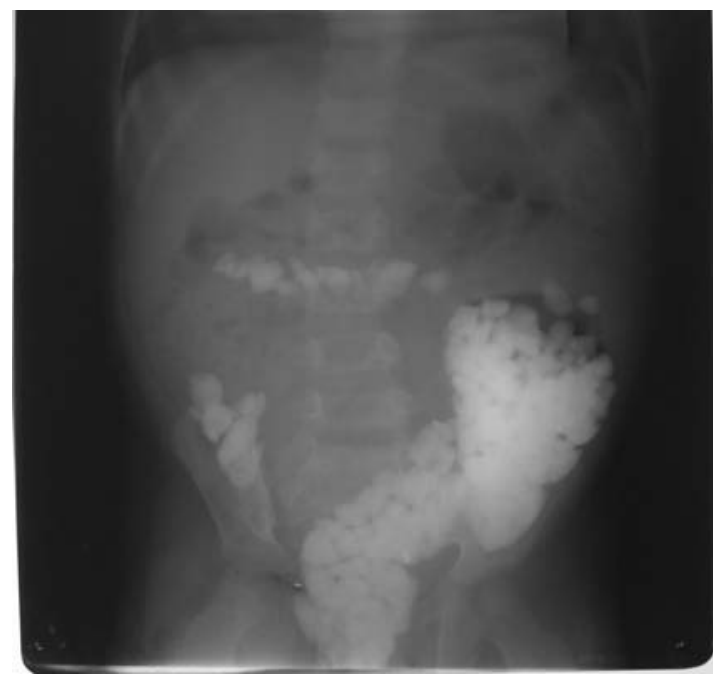

Fig. 1. X-ray abdomen in standing posture, showing multiple spherical radio-opaque shadows in ascending, transverse and descending colon.
Enteroliths are seen as multiple radio-opaque shadows of various shapes i.e, oval, round or rectangular with chronic abdominal symptoms. These shadows have dense rim with pale core of oval, round or rectangular features, coinend-on appearance and wide mobility. ${ }^{1}$ Enteroliths are intestinal calculi formed due to stasis and are known to occur with tuberculosis but has uncommonly been reported from India. ${ }^{4}$

Widespread abdominal lymph node calcification or diffuse peritoneal calcification due to abdominal tuberculosis also produce multiple radio-opaque shadows on plain abdominal radiograph. ${ }^{5,6}$ Abdominal lymph node calcification is occasionally caused by histoplasmosis or rarely by paracoccidioidomycosis and other chronic granulomatous infections. Fat nodule calcification, hydatid infestation, phlebolith formation in hemangiomatosis or remainants of radio-opaque dye may also produce similar picture on plain abdominal radiograph. $^{7}$

Sanjeev Kumar Digra, Virender Singh, Shirin Nomani ${ }^{1}$ and Varun Kaul

Department of Pediatrics, SMGS Govt. Medical College, Jammu, ${ }^{1}$ Department of Pediatrics, Apolo Hospital,

Bhubaneshwar, India.

E-mail : sanjeevahsaas@yahoo.co.in [DOI-10.1007/s12098-010-0089-y]

\section{REFERENCES}

1. Raidu BRS, Patnaik S, Rao CLV. Enteroliths. Ind I Radiol Imag 2002; 12: 135-136.

2. Uyemura Monte C. Foreign body ingestion in children. Am Fam Physician 2005; 72: 287-291.

3. Paul RI, Christoffel KK, Binns HJ et al. Foreign body ingestions in children : Risk of complication varies with site of initial health care contact. Pediatr 1993; 91: 121-127.

4. Mishra D, Singh S, Juneja M. Enterolithiasis: An uncommon finding in abdominal tuberculosis. Ind J Pediatr 2009; 76: 10491050.

5. McAfee JG, Donner MW. Differential diagnosis of calcifications encountered in abdominal radiographs. Am J Med Sci 1962; 118: 609-650.

6. Roy K. Diffuse peritoneal calcification - a rare manifestation of abdominal tuberculosis. Inter J Gynec and Obs 2009; 73: 269270 .

7. Khan MQ, Al-Qahtani AQ, Al-Momen S, Aldhurais SA, Ahmad M. Widespread tuberculous calcification. Saudi Med J 2000; 21: 386-389. 